Research Article

\title{
Dynamo Theory, Nonlinear Magnetic Fields, and the Euler Potentials
}

\author{
Bob Osano $\mathbb{D}^{1,2}$ \\ ${ }^{1}$ Cosmology and Gravity Group, Department of Mathematics and Applied Mathematics, University of Cape Town, \\ Rondebosch 7701, Cape Town, South Africa \\ ${ }^{2}$ Academic Development Programme, Science, Centre for Higher Education Development, University of Cape Town, \\ Rondebosch 7701, Cape Town, South Africa
}

Correspondence should be addressed to Bob Osano; bob.osano@uct.ac.za

Received 3 June 2018; Revised 30 August 2018; Accepted 20 September 2018; Published 10 October 2018

Academic Editor: Geza Kovacs

Copyright (C) 2018 Bob Osano. This is an open access article distributed under the Creative Commons Attribution License, which permits unrestricted use, distribution, and reproduction in any medium, provided the original work is properly cited.

\begin{abstract}
The debate on the role of Euler potentials (EP), $\alpha \nabla \beta$, in the dynamo theory is a long-standing one. It is known that the cross-product of gradients of two the potentials may represent magnetic fields lines. However, 2D and 3D dynamo hydromagnetic simulations suggest that their utility as analogues of magnetic field potential is restricted. This raises questions about their utility in the broader context of magneto-genesis and dynamo theories. We reexamine this and find that a reinterpretation of such potentials offers a new insight into the role EP may play in the general evolution of magnetic fields.
\end{abstract}

\section{Introduction}

Magnetic fields seem to permeate much of our visible universe. These fields are found in stars, galaxies, interstellar medium, and even clusters of galaxies. Such fields are detected via synchrotron emission, polarisation, or Faraday rotation in the radio frequency and appear between 0.2 and $10 \mathrm{GHz}$ [1]. Studies of cosmic magnetic fields have a long history and are driven by the need to explain what is observed and the desire to know what role magnetic fields play in the formation and evolution of cosmic structures ranging from stars and galaxies to superclusters and even the universe. We do not have a clear understanding of how all magnetic fields arise or how they evolve. Studies of cosmic magnetic fields are often classified as either (i) dealing with the origin, i.e., magneto-genesis, or (ii) dealing with growth or amplification, say, via a dynamo mechanism.

It is thought that original magnetic fields, or seed fields as they are commonly called, may have their origin in phase transition in the early universe [2,3], or in the cosmological structure formation [4], or in the first stars and black holes [5], or in the first supernova [6], or even as a result of fluctuations in the gravitational field [7]. The authors of [8] gave an argument that places severe constraints on magneto-genesis theories of the early universe. They provide proof that small-scale magnetic fields are unable to outlive the prerecombination era dominated by high resistivity due to recurrent electron-photon interactions. They arrive at the conclusion that cosmic magnetic fields generated before recombination had to have larger than the horizon coherence cell in order to survive recombination epoch or cosmic magnetic fields only emerging after recombination era. The explanation is that diffusion of magnetic fields is hampered by distances greater than the horizon which forces the cells to become subhorizon after recombination. Whichever way magneto-genesis occurs, the resulting fields need sustaining for them to be detectable. This is thought to be primarily via a dynamo action $[9,10]$.

This article deals mainly with dynamo effect and Euler potentials. Research on dynamo action has a long history that goes back to the work of [11] where a homogeneous dynamo mechanism was proposed as a possible source of magnetic fields observed in the sunspots. The proposal remained largely ignored particularly because of the results published soon after in [12] which showed that it was impossible to generate axisymmetric or two-dimensional magnetic fields via the homogeneous dynamo process. This became known as Cowling's theorem. It was not until after $[13,14]$ were published 
that the ideas in [11] were salvaged. Whereas Cowling showed that it was not possible to generate axisymmetric magnetic fields, the restriction did not apply to the nonaxisymmetric ones. Backus [13] and Herzenberg [14] demonstrated that nonaxisymmetric fields could indeed be generated via a homogeneous dynamo. In effect, they presented arguments for the threshold necessary for the dynamo action. These and other works on the threshold are classified as bounding theorems as they provide lower bounds for this kind of dynamo. The questions then changed from whether or not a dynamo action could take place to what threshold was needed to induce the action.

The dynamo term in the magnetic induction equation is the term $\nabla \times(\mathbf{U} \times \mathbf{B})$, where $\mathbf{U}$ is the velocity and $\mathbf{B}$ is the magnetic flux. It is clear that the larger this term, the greater the amplification of the magnetic field. Different forms of bounding theorems appear in literature giving special cases and designed for specific problems. In general, the velocity field has to be strong enough in order to stretch the magnetic fields to the point where they overcome Ohmic dissipation [13, 15-20]. Most of these analyses are mathematical, but mathematical analysis alone without experimentation can only yield a limited understanding of the dynamo mechanism. On the other hand, the likelihood of replicating some of the extreme conditions needed for dynamos is slim. Computer simulations have emerged as a powerful alternative and one that is contributing to greater understanding dynamos. Simulations also offer a platform for examining magnetic analogues [21-24] or testing predictions that result from any new formulation of electrodynamics, for example, the formulation given in [25]. In the remaining sections, we revisit the theoretical basis for the dynamo action and comment on Euler potentials in relation to the evolution of magnetic fields. Simulations related to Euler potentials have recently appeared [26-28].

\section{Induction Equation: The Standard Approach}

The starting point for most discussions of magnetic induction is the set of Maxwell's equations, which in MKS units are

$$
\begin{gathered}
\frac{\partial \mathbf{B}}{\partial t}=-\nabla \times \mathbf{E}, \quad \nabla \cdot \mathbf{B}=0 \\
\epsilon_{0} \mu_{0} \frac{\partial \mathbf{E}}{\partial t}=\nabla \times \mathbf{B}-\mu_{0} \mathbf{J}, \quad \nabla \cdot \mathbf{E}=\frac{4 \rho}{\epsilon_{0}}
\end{gathered}
$$

where $\mathbf{E}$ is the electric field, $\mathbf{J}$ is the current density, and $\mu 0$ is the magnetic permeability such that the permittivity of free space $\epsilon_{0}=1 / c^{2} \mu_{0}$.

In the present context, a further ingredient necessary for establishing the primary equation is Ohm's law. Assuming a plasma fluid, the standard form of this law has the structure

$$
\mathbf{E}+\mathbf{U} \times \mathbf{B}=\eta J+\frac{1}{n e} \mathbf{J} \times \mathbf{B}-\frac{1}{n e} \nabla \boldsymbol{p},
$$

where we have neglected the time variation of the current density $\partial \mathbf{J} / \partial t . \eta$ is the magnetic diffusivity and expresses the relationship between electric field, moving charges of a given density and a magnetic flux. $n$ is the electron number density and $e$ is the charge. Now consider the case where the Hall's term $(\mathbf{J} \times \mathbf{B} /$ ne $)$ may be neglected and where the gradient of pressure term may be replaced by the product of a scalar potential and the gradient of a different scalar potential, a Euler potential term (we will return to this correspondence later in the article). The standard approach is to choose a time scale in which the displacement current can be neglected. This implies that the time derivative of the electric field is set to zero leading to $\nabla \times \mathbf{B}=\mathbf{J}$ and since $E=-\mathbf{U} \times \mathbf{B}+\eta \mathbf{J}$ from our modified Ohm's law, it is easy to see that the evolution equation for the magnetic flux is given by the following.

$$
\frac{d \mathbf{B}}{d t}=\nabla \times(\mathbf{U} \times \mathbf{B}-\eta \nabla \times \mathbf{B} .)
$$

The $\mathbf{U} \times \mathbf{B}$ is the usual induction term. Traditionally, the velocity term stretches the field thereby enhancing its strength. The useful form of (17) is as follows.

$$
\frac{d \mathbf{B}}{d t}=\nabla \times(\mathbf{U} \times \mathbf{B})+\eta \nabla^{2} \mathbf{B}
$$

Let us now consider the modification of the induction equation.

\section{Induction Equation and Euler Potentials}

The Coulomb gauge is the cornerstone of Maxwell equations and we need to understand it in relation to vector potentials. In particular, the magnetic vector potential $\mathbf{A}$ (hereafter $\mathbf{A}_{M P}$ ) is chosen such that it obeys the Coulomb gauge; i.e., $\nabla \cdot \mathbf{A}_{M P}=$ 0 ; otherwise one can add a gradient of any scalar $\nabla \Xi$ to obtain a variation of the vector potential $\mathbf{A}^{!}=\mathbf{A}_{M}+\nabla \Xi$. The problem here lies in the fact that $\nabla \times \mathbf{A}^{!}=\nabla \times \mathbf{A}_{M P}=\mathbf{B}$. Any spurious gauge function such as $\Xi$ does not lead to a magnetic field but has the consequence that $\nabla \cdot \mathbf{A}^{!} /=0$, other than special cases as we shall demonstrate. Therefore, let us go back to the main point of this article which has to do with the dynamo equation in the presence of scalar functions. A good starting point is with Euler potentials [26, 29, 30]. It was argued in [26] that a magnetic field $\mathbf{B}$, which we denote by $\mathbf{B}_{E P}$, could be represented by the Euler potentials denoted by $(\alpha, \beta)$ such that

$$
\nabla \alpha \times \nabla \beta \equiv \mathbf{B}_{E P}
$$

with the consequence that every magnetic field line would be labeled by these two variables. A variation of these potentials with respect to time provides a velocity $\mathbf{U}$ representing the movement of magnetic field lines with the consequence that a particle moving with this velocity maintains the properties of the potentials. In particular,

$$
\begin{aligned}
& \frac{\partial \alpha}{\partial t}+\mathbf{U} \cdot(\nabla \alpha)=0 \\
& \frac{\partial \beta}{\partial t}+\mathbf{U} \cdot(\nabla \beta)=0 .
\end{aligned}
$$


Since the direction of motion is perpendicular to the magnetic field line, U.B. $\mathbf{B}_{E P}=0$. Multiplying (7) by $\nabla \beta$ and (8) by $\nabla \alpha$, subtracting the resulting equation, and applying the vector identity $\nabla \beta(\mathbf{U} . \nabla \alpha)-\nabla \alpha(\mathbf{U} . \nabla \beta)=\mathbf{U} \times(\nabla \alpha \times \nabla \beta)$, the author of [26] showed the following.

$$
\mathbf{U} \times(\nabla \alpha \times \nabla \beta)=\nabla \alpha \frac{\partial \beta}{\partial t}-\nabla \beta \frac{\partial \alpha}{\partial t}
$$

Taking the curl of (9) and using a vector identity, they were able to show that

$$
\frac{\partial B_{E P}}{\partial t}=\nabla \times\left(U \times B_{E P}\right)
$$

which is the dynamo term in the induction equation but also give the definition of field like velocity [31-33]. This amounts to the conjecture on existence of fast dynamos, where the field growth is not dependent on diffusion. However, as shown in [34], conservation of magnetic helicity severely restricts fast dynamos. For a more general discussion, we seek a formulation that includes diffusivity. The Euler scalars are conserved along field lines such that they allow for the description of magnetic field lines. The field lines can be thought of as given by the intersections of two surfaces defined by the gradients of the potentials. In particular, we define two scalar potential functions $\alpha$ and $\beta$ with the properties $\nabla \alpha . \mathbf{B}_{E P}=0=\nabla \beta \cdot \mathbf{B}_{E P}$. In effect, both gradient scalars are orthogonal to the field lines and in addition are orthogonal to each other such that

$$
\nabla \alpha \times \nabla \beta=\zeta \mathbf{B}_{E P},
$$

where the coefficient $\zeta$ is a pure scalar quantity which may depend on time. This looks structurally like the $\alpha$-effect in the mean-field theory [35]. Another way of looking at this is that we have a vector potential function $\mathbf{A}_{E P}=\alpha \nabla \beta$, which allows the definition

$$
\zeta \mathbf{B}_{E P}=\nabla \times \mathbf{A}_{E P}=\nabla \times(\alpha \nabla \beta)=\nabla \alpha \times \nabla \beta .
$$

Of course, one would like to know how this affects gauge choice. To be specific, we would like to know if this new vector potential function, $\alpha \nabla \beta$, obeys the Coulomb gauge condition and if not what physically motivated restrictions would lead to it. The divergence of the new scalar potential function is as follows.

$$
\nabla \cdot \mathbf{A}_{E P}=\nabla \cdot(\alpha \nabla \beta)=\nabla \alpha \cdot \nabla \beta+\alpha \nabla^{2} \beta
$$

We need to consider divergence theorem; to be more explicit we need the Green's identity of the first kind, in order to resolve the right-hand side of this equation. On applying divergence theorem to the $A_{M P}=\alpha \nabla \beta$, where $\beta$ is twice differentiable, we obtain

$$
\begin{aligned}
\iiint_{V} \nabla \cdot(\alpha \nabla \beta) d V & =\iiint_{V}\left(\alpha \nabla^{2} \beta+\nabla \alpha \cdot \nabla \beta\right) d V \\
& =\iint_{C} \alpha \nabla \beta \cdot d S
\end{aligned}
$$

where $V$ is a typical volume and $C$ the boundary of enclosing $V$. The special subcase of (14) is when $\nabla \beta$ is orthogonal to the oriented surface $\mathbf{S}$. This, together with the fact that the volume $V$ can be made arbitrarily small, leads to $\nabla \cdot(\alpha \nabla \beta)=0$. We note that in general $\nabla \beta . d \boldsymbol{S} \neq 0$. Given our orthogonal requirement, we now have a vector potential function that is defined by two scalar functions. Clearly, the rate at which the magnetic vector potential $\mathbf{A}_{M P}$ grows will be modified to

$$
\frac{d \mathbf{A}_{M P}}{d t}=\mathbf{U} \times\left(\nabla \times \mathbf{A}_{M P}\right)+\mathbf{A}_{E P}+\eta \nabla \mathbf{A}_{M P}-\nabla \varphi
$$

where $\eta$ is the coefficient of diffusion and $\varphi$ is any general scalar potential function. Taking the curl of (15) and using relevant vector identities, we get

$$
\frac{d \mathbf{B}}{d t}=\nabla \times(\mathbf{U} \times \mathbf{B})+\eta \nabla^{2} \mathbf{B}+(\nabla \alpha \times \nabla \beta)
$$

where again $\mathbf{B}=\nabla \times \mathbf{A}_{M P}$. It is clear that this equation can be derived beginning with the idea that charges experience the effect of a vector potential $\alpha \nabla \beta$ leading to a modified Ohm's law of the form $E+\mathbf{U} \times \mathbf{B}+\alpha \nabla \beta=\eta \mathbf{J}$. Again, a time scale is chosen allowing the displacement current to be neglected. It is easy to show that form of this equation leads to an evolution equation for the magnetic flux given by the following.

$$
\frac{d \mathbf{B}}{d t}=\nabla \times(\mathbf{U} \times \mathbf{B}+\alpha \nabla \beta-\eta \nabla \times \mathbf{B} .)
$$

The $\mathbf{U} \times \mathbf{B}$ is the usual induction term. It would appear that the term $\alpha \nabla \beta$ plays a role complementary to the inductive term. The velocity term is known to stretch the field thereby enhancing its strength. In contrast, the Euler term seems to affect the field via the cross-product of the gradients of the two scalars. Could this geometric effect lead to an enhancement of the field? Indeed, it is the role that this term plays that we seek to understand. The useful form of (17) is as follows.

$$
\frac{d \mathbf{B}}{d t}=\nabla \times(\mathbf{U} \times \mathbf{B})+\eta \nabla^{2} \mathbf{B}+(\nabla \alpha \times \nabla \beta)
$$

Recent developments related to Euler potentials are worth pointing out. In [27], $\nabla \times \mathbf{A}_{M P}$ is compared to $\nabla \times \mathbf{A}_{E P}$ where artificial viscosity is used in simulations involving the latter.

This follows the extensive application of $A_{E P}$ in the SPH approach $[36,37]$ to structure formation. It is found in [27] that the $\nabla \times \mathbf{A}_{E P}$ compares with $\nabla \times \mathbf{A}_{M P}$ when $\eta=0$ and that the introduction of $\eta /=0$ leads to discrepancies. The conclusion is that $\mathbf{A}_{E P}$ and $\mathbf{A}_{M P}$ cannot be used interchangeably, in particular that the growth of $\nabla \times \mathbf{A}_{E P}$ cannot be ascribed to a dynamo effect. Be that as it may, (17) suggests that $\mathbf{A}_{E P}$ may play a role in the amplification of $\nabla \times \mathbf{A}_{M P}$, which is not in contradiction to [27]. But what other role might this potential play? In studies of the self-consistent or the dynamic dynamo problem, the momentum equation provides the necessary ingredient for a time varying velocity. In particular,

$$
\rho \frac{D \mathbf{U}}{D t}=-\nabla p+\mathbf{J} \times \mathbf{B}+\sum f+F_{v i s c}
$$

where $D / D t=\partial / \partial t+\mathbf{U} \cdot \nabla$ is the Lagrangian derivative. $\mathbf{U}$ is the bulk velocity of the fluid, $\rho$ is fluid density, $p$ is fluid 
pressure, and $\mathbf{F}_{\text {visc }}$ is fluid viscous force, while $\Sigma f$ is the sum of all body forces acting on the fluid. Equations (17) and (19), with various modifications and physical considerations, usually form the basis of most magnetic dynamo studies. We investigate the usefulness of (18) and (19).

\section{Particle Drift in Ionosphere-Magnetosphere Coupling: Application}

From the discussion above, it appears that EP representation could be used as the analogue of magnetic fields. Indeed, this is advantageous for several reasons. Firstly, the representation is automatically divergence-free $(\nabla \cdot(\nabla \times \alpha \nabla \beta)=0)$ and, secondly, the field lines can be directly visualized. These advantages have led to new understanding of magnetic fields. Consider the case where Hall's term is nonnegligible $(\mathbf{J} \times$ $\mathbf{B} / \mathbf{~ = 0 )}$ due to a nonvanishing Lorentz force in the momentum equation. In their discussion of mathematical modelling of magnetic magnetospheric convection and its coupling to the ionosphere, the authors of [38] showed that the momentum conservation equation for slowly moving plasma, in comparison to the thermal speed, is given by

$$
\nabla_{p}=\mathbf{J} \times \mathbf{B},
$$

where the convention $c=1$ has been used. This is clearly the case as can be seen from (19) when all body and viscous forces are neglected, and the velocity is constant. Taking the cross of $\mathbf{B}$ with (20) and requiring that J.B $=0$ yield the isotropic orthogonal component of the current density.

$$
\mathbf{J}_{\perp}=\frac{\mathbf{B} \times \nabla p}{B^{2}}
$$

As discussed in [38] the current density in (21) is in general not divergence-free. The total current has to be divergencefree. It is easy to show (as was done in [38]) that under magnetospheric constraints the charge accumulation resulting from the divergence of the right-hand side of (21) leads to an electric current that changes on the timescale similar to that of the displacement current in Maxwell's equations. Mathematically,

$$
\nabla \cdot J_{\perp}=\frac{(\mathbf{B} \times \nabla B) \cdot \nabla p}{B^{3}}+\frac{\nabla p \cdot(\nabla \times \mathbf{B})}{B^{2}}-\frac{\mathbf{B} \cdot \nabla \times p}{B^{2}}
$$

the last term is clearly zero from vector identities, while the second term on the right vanishes when the pressure gradient is perpendicular to $\nabla \times \mathbf{B}$. This leads to Vasyliunas' convective current equation representing the derivative of $\mathbf{J}_{\|}$along the magnetic field direction and is given by the following.

$$
\frac{\partial}{\partial l}\left(\frac{\mathbf{J}_{\|}}{B}\right)=\frac{2(\mathbf{B} \times \nabla B) \cdot \nabla p}{B^{4}}
$$

This equation provides a computational scheme for examining electrodynamics in the coupling of ionosphere and magnetosphere. The authors of [39] have used a variant of this equation; namely,

$$
\left(\frac{\mathbf{J}_{\| n}}{B_{n}}-\frac{\mathbf{J}_{\| s}}{B_{s}}\right)=\frac{\boldsymbol{b} \cdot(\nabla V \times \nabla p)}{B}
$$

where $J_{\| n}$ and $J_{\| s}$ are the Birkeland current densities along the magnetic field direction in the north and the south ionosphere. $\boldsymbol{b}$ is a unit vector along the magnetic field line, while $V=d s / B$ is the volume of a unit magnetic flux tube and $p$ is the magnetospheric particle pressure. Implementing (24) in computer simulations is greatly aided by adopting the EP as the ansatz for the magnetic field. In this regard, substituting (11) into (24) yields the easier-to-implement scalar expression

$$
\left(\frac{\mathbf{J}_{\| n}}{B_{n}}-\frac{\mathbf{J}_{\| s}}{B_{s}}\right)=\frac{\partial V}{\partial \alpha} \frac{\partial p}{\partial \beta}-\frac{\partial p}{\partial \alpha} \frac{\partial V}{\partial \beta},
$$

where the right-hand side can be evaluated anywhere along the field line. Some comment is required about the use of Euler potentials in this approximation. In this case (11) is used as an analogue of magnetic field flux leading to the substitution. This differs from our treatment where EP does not replace B but contributes to it via the modified Ohm's law. Nevertheless, the form that is given in (24) allows one to study magnetosphere-ionosphere coupling as was demonstrated in [39]. There are other areas where EP has proved to be useful in studies of $\mathrm{x}$-shape magnetic fields [40]. There will undoubtedly be more areas where EP are used and therefore a continued examination of EP is useful. We have seen that EP can be used as ansatz for magnetic field flux, but this has a limitation as pointed out in [27, 41]. We have introduced EP via the modified Ohm's law. We want to further examine this.

\section{Pressure, Density, and Euler Potentials}

Returning to (14) and the ensuing discussion, $\alpha \nabla \beta$ looks structurally like $\nabla p / \rho$ (the Biermann battery term) suggesting the correspondence:

$$
\nabla \alpha \times \nabla \beta=\frac{\nabla \mathrm{p} \times \nabla \rho}{\rho^{2}}
$$

where $p$ and $\rho$ are electron pressure and density, respectively. The implied correspondence in (26) calls for further analysis. It is suggestive of the EP potential role in momentum (19), probably coming in as a body force divided by the density. It is conceivable that in an idealized fluid flow, density and pressure may exhibit properties similar to Euler potentials and thus raising the prospect of more complex interactions between fluid and magnetic field lines, over and above the back reaction via Lorentz force. However, (17) and correspondence (26) show that if the position of the two gradients of scalars is switched, this term acts as a diffusive term. This may partly account for the results obtained in [42]. We have only dealt with a simplified case of fluid flow where the Lorentz force is switched off because our interest is in examining how Euler potentials affect the threshold for dynamo action and not assessing the long-term contribution to the evolution of magnetic fields.

\section{Discussion and Conclusion}

In this rather inquisitive article, we have delved into the subject of the dynamo theory. Our interest was piqued by the need to reexamine the basis for dynamo theory in a 
flow where Euler potentials are present. We have done four things. (1) In deriving the induction equation, we find that the following form of Ohm's law $\mathbf{E}=-\mathbf{U} \times \mathbf{B}+\eta \mathbf{J}-\alpha \nabla \beta$ is inevitable.

The $\alpha \nabla \beta$ acts as a source for electric currents and needs further investigations. (2) We have also shown that electron density and pressure variables are natural candidates for Euler potentials for flows in which $\nabla p$ and $\nabla \rho$ are conserved but not parallel. In fact, the $\nabla p / \rho$ is itself a vector potential from which magnetic flux originates. The curl of this vector potential is the basis for Biermann battery term in magnetogenesis. (3) In the appendix, we show that Backus theorem for magnetic induction holds even when Euler potentials are considered. (4) We find that indeed the strength of the strain tensor required for the dynamo action to kick in would be lower when the effect of such potentials is considered. Simulations comparing the EP method and the A approaches were performed in [27], where it was found that two potentials exhibit different growth patterns when diffusivity was included. This suggests that the two potentials are not interchangeable but complementary.

\section{Appendix}

\section{Energetics and the Dynamo Action}

The concept of a dynamo is that it is a mechanism that allows for kinetic energy in a system to be converted into magnetic energy [10]. One presupposes that such a system is isolated, and the growth of magnetic energy is attributed to the mechanism and not to an external contributor. The magnetic energy of such a system is given by

$$
\varepsilon_{m}=\iiint \frac{1}{2 \mu}|\mathbf{B}|^{2} d V .
$$

Our interest is in how magnetic energy changes, more specifically increases, with respect to time. In order to estimate the change in magnetic energy, we take the derivative of (A1) with respect to the time parameter and express it as follows:

$$
\mu \frac{d \varepsilon_{m}}{d t}=\iiint_{V} \mathbf{B} \cdot \frac{d \mathbf{B}}{d t} d V
$$

where the right-hand side is an integral over a volume $V$ of finite conductivity. The term $d \mathbf{B} / d t$ may then be eliminated using magnetic induction (17) to give

$$
\begin{gathered}
\mu \frac{d}{d t}\left(\iiint_{2} \frac{1}{2}|\mathbf{B}|^{2} d V=\iiint_{V} \mathbf{B} \cdot(\nabla \times(\mathbf{U} \times \mathbf{B})) d V\right) \\
+\eta \iiint_{V} \mathbf{B} \cdot\left(\nabla^{2} \mathbf{B}\right) d V+\varsigma \iiint_{V} \mathbf{B} \cdot \mathbf{B} d V
\end{gathered}
$$

and note that one can expanded the first term on the righthand side of (17) into the constituent terms: the stretching, the advection, and the compression, where we have assumed that the magnetic flux is homogeneous. We also used definition (11) in our substitution to obtain the last term in (17). The magnitude of the terms of on the right-hand side of this equation has to be positive. Several treatments appear in literature of ways of assessing this. We use Backus approach [13]. Following this formulation, we use vector manipulations to show that $\mathbf{B} \cdot\left(\nabla^{2} \mathbf{B}\right)$ is equivalent to $|\nabla \times \mathbf{B}|^{2}$. In order for a dynamo to work, the diffusive term must be significantly less than the amplifying term. How significantly less should diffusion be? this can be answered by determining a suitable scale for making quantitative comparison. To this end we define the following parameters:

$$
\begin{aligned}
m(t) & =\max \left(d_{i} u_{j}+d_{j} u_{i}\right) \\
s_{d} & =\min \left(\frac{\varsigma \int_{V} \mathbf{B} \cdot\left(\nabla^{2} \mathbf{B}\right) d V}{(d / d t) \int_{V^{\prime}}|\mathbf{B}|^{2} d V}\right) \\
f_{d} & =\min \left(\frac{\eta \int_{V} \mathbf{B} \cdot \mathbf{B} d V}{(d / d t) \int_{V^{\prime}}|\mathbf{B}|^{2} d V}\right)
\end{aligned}
$$

where $m(t)$ is the maximum of the rate of strain tensor. This leads to the modified inequality.

$$
\begin{aligned}
& \frac{1}{2} \frac{d}{d t} \iint_{V+V^{\prime}}|\mathbf{B}|^{2} d V \\
& \quad \leq\left(m(t)+f_{d}-s_{d}\right) \frac{d}{d t} \iint_{V+V^{\prime}}|\mathbf{B}|^{2} d V
\end{aligned}
$$

$V+V^{!}$indicates an integral over the entire space, whereas $V^{!}$ only covers a part of $V$ and arises from the partial integration of the term $\nabla \times(\eta \nabla \times \mathbf{B})$. Dynamo action occurs as long as the net effect of the stretching $m(t)$ and the focusing $f d$ is greater than the dissipative term $s d$; i.e., $m(t)+f d>s d$. The implication is that the maximum value of the strain tensor need not be as high as previously thought if focusing is taken into account.

\section{Data Availability}

No data were used to support this study.

\section{Conflicts of Interest}

The author declares that they have no conflicts of interest.

\section{Acknowledgments}

The author acknowledges the support from the University of Cape Town's URC and NGP funds.

\section{References}

[1] R. Beck, A. Brandenburg, D. Moss et al., "Galactic Magnetism: Recent Developments and Perspectives," Annual Reviews, vol. 34, pp. 155-206, 1996.

[2] C. Caprini, R. Durrer, and E. Fenu, "Can the observed large scale magnetic fields be seeded by helical primordial fields?" Journal of Cosmology and Astroparticle Physics, vol. 2009, no. 11, 2009.

[3] L. M. Widrow, "Origin of galactic and extragalactic magnetic fields," Reviews of Modern Physics, vol. 74, no. 3, pp. 775-823, 2002. 
[4] M. Lazar, R. Schlickeiser, R. Wielebinski, and S. Poedts, "Cosmological effects of weibel-Type instabilities," The Astrophysical Journal, vol. 693, no. 2, pp. 1133-1141, 2009.

[5] M. J. Rees, Cosmic Magnetic Fields, R. Wielebinski and R. Beck, Eds., Springer, Berlin, Germany, pp.1-8, 2005.

[6] H. Hanayama, K. Takahashi, K. Kotake, M. Oguri, K. Ichiki, and H. Ohno, "Biermann mechanism in primordial supernova remnant and seed magnetic fields," The Astrophysical Journal, vol. 633, no. 2 I, pp. 941-945, 2005.

[7] B. Mongwane, P. K. Dunsby, and B. Osano, "Cosmic electromagnetic fields due to perturbations in the gravitational field," Physical Review D: Particles, Fields, Gravitation and Cosmology, vol. 86, no. 8, 2012.

[8] H. Lesch and G. T. Birk, "Can large-scale magnetic fields survive during the pre-recombination era of the universe?" Physics of Plasmas, vol. 5, no. 7, pp. 2773-2776, 1998.

[9] R. Beck, "Cosmic Magnetic Fields," in Proceedings of the AIP Conference, vol. 1381, 2011.

[10] A. Brandenburg and K. Subramanian, "Astrophysical magnetic fields and nonlinear dynamo theory," Physics Reports, vol. 417, no. 1-4, pp. 1-209, 2005.

[11] J. Larmor, "How could a rotating body such as the sun become a magnet?" Report of the British Association for the Advancement of Science, pp. 159-160, 1919.

[12] T. G. Cowling, “The Magnetic Field of Sunspots," Monthly Notices of the Royal Astronomical Society, vol. 94, no. 1, pp. 3948, 1933.

[13] G. Backus, "A class of self-sustaining dissipative spherical dynamos," Annals of Physics, vol. 4, pp. 372-447, 1958.

[14] A. Herzenberg, "Geomagnetic dynamos," Philosophical Transactions of the Royal Society of London. Series A. Mathematical and Physical Sciences, vol. 250, pp. 543-583, 1958.

[15] S. Childress, Theorie magnetohydrodynamique de l'effet dynamo Lecture notes, Departement Mechanique de la Faculte des Science, Paris, France, 1969.

[16] M. R. Proctor, “On Backus' necessary condition for dynamo action in a conducting sphere," Geophysical \& Astrophysical Fluid Dynamics, vol. 9, no. 1, pp. 89-93, 1977.

[17] M. R. E. Proctor, "Necessary Conditions for the Magnetohydrodynamic Dynamo," Geophysical \& Astrophysical Fluid Dynamics, vol. 14, no. 1, pp. 127-145, 1979.

[18] A. P. Willis, "Optimization of the Magnetic Dynamo," Physical Review Letters, vol. 109, no. 25, 2012.

[19] B. M. Byington, N. H. Brummell, J. M. Stone, and D. O. Gough, "Stoked nondynamos: sustaining field in magnetically nonclosed systems," New Journal of Physics, vol. 16, no. 8, p. 083002 , 2014.

[20] L. Chen, W. Herreman, and A. Jackson, "Optimal dynamo action by steady flows confined to a cube," Journal of Fluid Mechanics, vol. 783, pp. 23-45, 2015.

[21] B. Osano and P. Adams, "Analogue Magnetism Revisited," https://arxiv.org/abs/1602.09105.

[22] B. Osano and P. W. Adams, "Toward the analogue of a thermally generated electromagnetic field," Journal of Mathematical Physics, vol. 58, no. 9, 093101, 10 pages, 2017.

[23] B. Osano and P. Adams, "Technical considerations in Magnetic Analogue Models," https://arxiv.org/abs/1606.06725.

[24] B. Osano and P. Adams, "Analogue Magnetism: An Ansatz," https://arxiv.org/abs/1607.00980.

[25] B. Osano, "Generalised Maxwellian Equations and their Implications," https://arxiv.org/abs/1604.00263.
[26] D. Stern, "Euler potentials and geomagnetic drift shells," Journal of Geophysical Research: Atmospheres, vol. 73, no. 13, pp. 43734378, 1968.

[27] A. Brandenburg, "Magnetic field evolution in simulations with Euler potentials," Monthly Notices of the Royal Astronomical Society, vol. 401, no. 1, pp. 247-354, 2010.

[28] K. Dolag and F. Stasyszyn, "An MHD gadget for cosmological simulations," Monthly Notices of the Royal Astronomical Society, vol. 398, no. 4, pp. 1678-1697, 2009.

[29] L. Euler, Sectio Secunda de Principiis Motus Fluidorum Novi Comentarii Acad. Sci. Petropolitanae 4,270 (1769); reprinted in Leonhardi Euleri Opera Omnia, Series II, Vol.13, pp. 73, published by the Swiss Society for Natural Sciences, 1955.

[30] C. Truesdell, The Kinematics of Vorticity, Indiana University Publications Science Series no. 19, Indiana University Press, Bloomington, 1954.

[31] D. Stern, "The motion of magnetic fieldlines," Space Science Reviews, vol. 6, no. 2, pp. 147-173, 1966.

[32] W. A. Newcomb, "Motion of magnetic lines of force," Annals of Physics, vol. 3, pp. 347-385, 1958.

[33] T. G. Northrop, "Adiabatic charged-particle motion," Reviews of Geophysics, vol. 1, no. 3, 1963.

[34] H. K. Moffatt and M. R. E. Proctor, "Topological constraints associated with fast dynamo action," Journal of Fluid Mechanics, vol. 154, pp. 493-507, 1985.

[35] A. Hubbard, F. Del Sordo, P. J. Käpylä, and A. Brandenburg, "The $\alpha$ effect with imposed and dynamo-generated magnetic fields," Monthly Notices of the Royal Astronomical Society, vol. 398, no. 4, pp. 1891-1899, 2009.

[36] D. J. Price and J. J. Monaghan, "Smoothed Particle Magnetohydrodynamics - III. Multidimensional tests and the $\nabla \cdot \mathrm{B}=0$ constraint," Monthly Notices of the Royal Astronomical Society, vol. 364, no. 2, pp. 384-406, 2005.

[37] D. J. Price and M. R. Bate, "The impact of magnetic fields on single and binary star formation," Monthly Notices of the Royal Astronomical Society, vol. 377, no. 1, pp. 77-90, 2007.

[38] V. M. Vasyliunas, "Mathematical Models of Magnetospheric Convection and its Coupling to the Ionosphere," in Particles and Fields in the Magnetosphere, B. M. McCormac, Ed., vol. 17 of Astrophysics and Space Science Library, pp. 60-71, Springer, New York, NY, USA, 1970.

[39] R. A. Wolf, R. W. Spiro, S. Sazykin, F. R. Toffoletto, P. Le Sager, and T. Huang, "Use of Euler potentials for describing magnetosphere-ionosphere coupling," Journal of Geophysical Research: Atmospheres, vol. 111, no. A7, 2006.

[40] K. Ferriere and P. Terral, "Analytical models of X-shape magnetic fields in galactic halos," Astronomy \& Astrophysics, vol. 561, no. A100, 2014.

[41] O. A. Reula and M. E. Rubio, "Ill posedness of force-free electrodynamics in Euler potentials," Physical Review D: Particles, Fields, Gravitation and Cosmology, vol. 95, no. 6, 064005, 14 pages, 2017.

[42] A. Brandenburg, "Simulations of astrophysical dynamos," in Advances in Plasma Astro- physics, ed. Proc. IAU, A. Bonanno, E. de Gouveia dal Pino, and A. Kosovichev, Eds., vol. 6, IAU Symp. S274, pp. 402-409, 2010. 

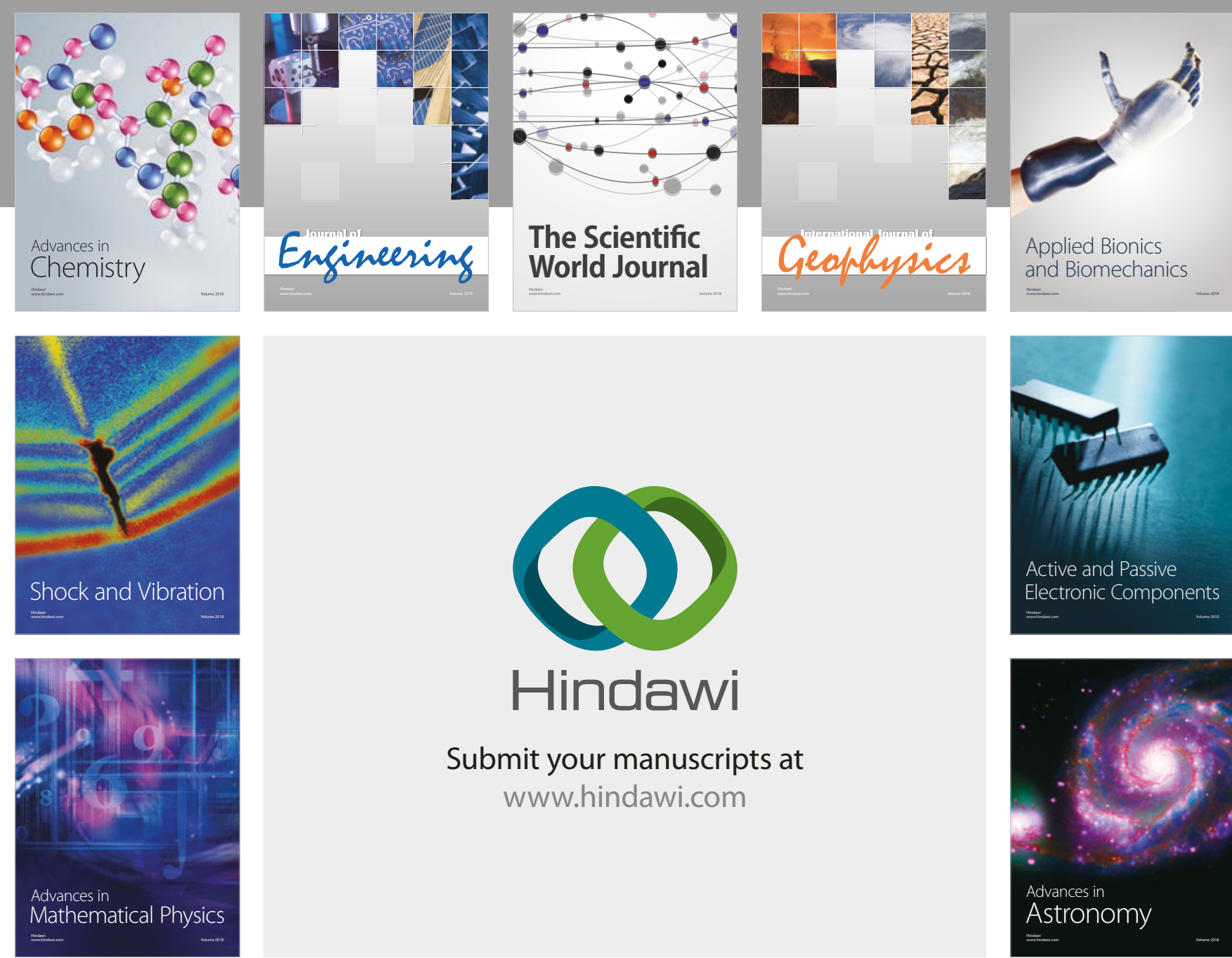

Submit your manuscripts at

www.hindawi.com

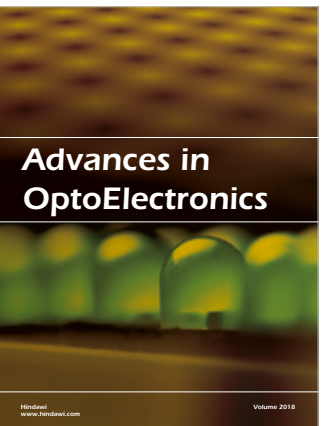

\section{Rotcting Machinery}
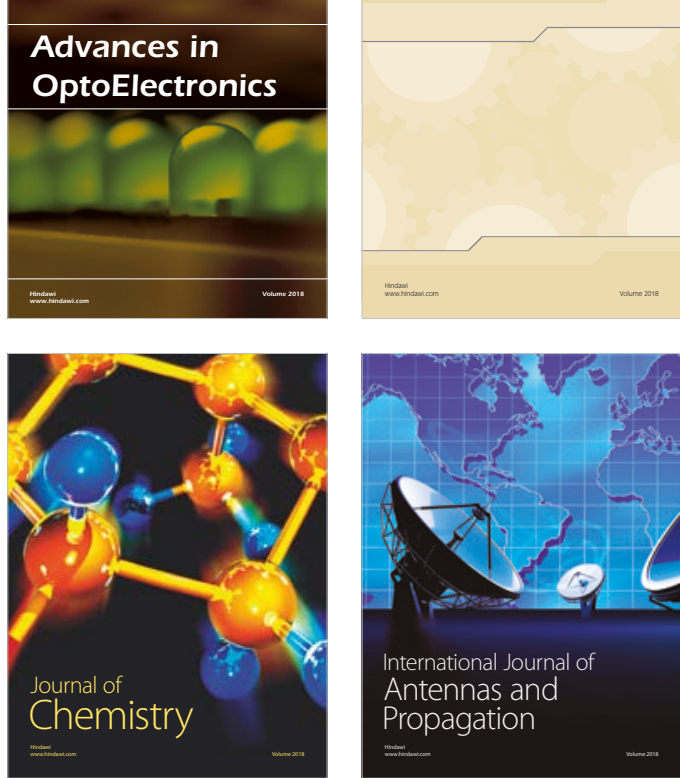

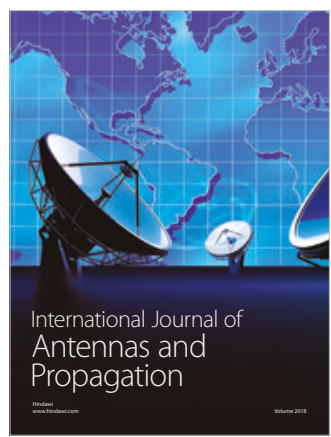

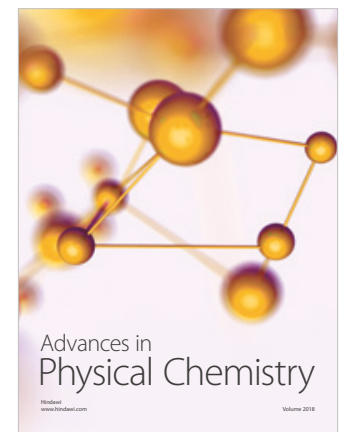

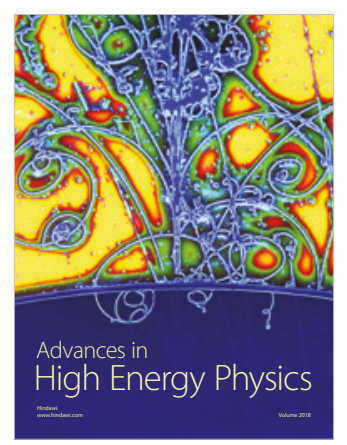

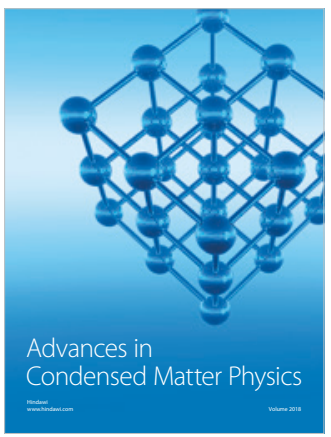

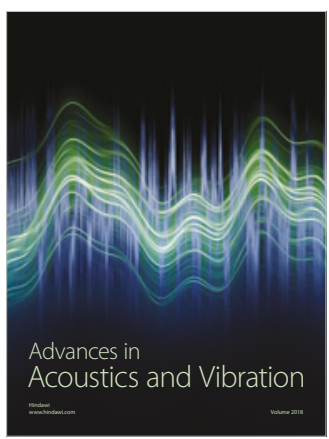

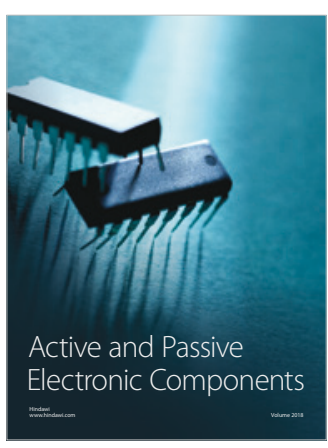
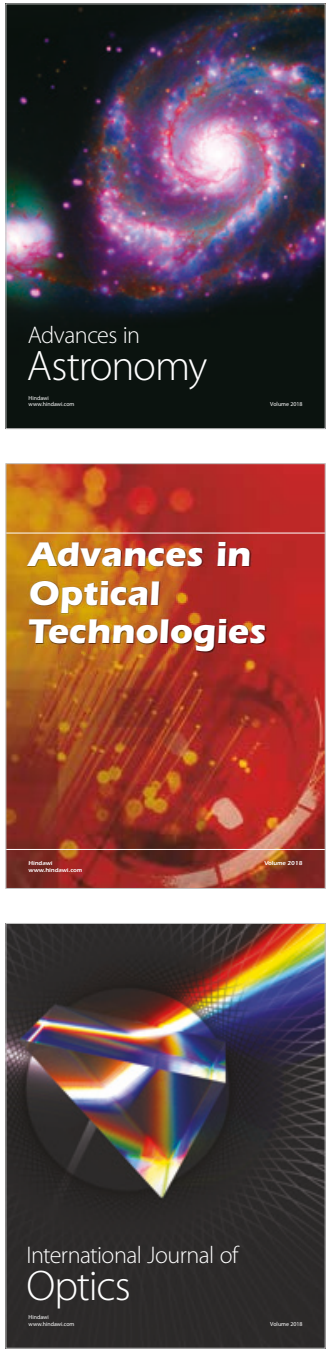